Rev. Saúde píbl., S. Paulo

9:441-54, 1975.

\title{
AVALIAÇÃO PRELIMINAR DO SERVIÇO DE SAÚDE ESCOLAR DA IX REGIÃO ADMINISTRATIVA, RIO DE JANEIRO: ESTUDO DA POPULAÇÃO COBERTA E ANALISE OPERACIONAL DE UM SUBPROGRAMA *
}

\author{
Eneida Duarte Gaspar ** \\ Márcio José de Almeida ** \\ José Augusto A. C. Taddei ** \\ Eliana Cláudia O. Ribeiro** \\ Sandra da Silva Pereira*** \\ Maria Cristina $\mathrm{T}$. Vilanova** \\ Hésio de Albuquerque Cordeiro** \\ Jeiel C. Ferreira de Souza ****
}

RSPU-B/283

GaSPaR, E. D. et al. - Avaliação preliminar do Serviço de Saúde Escolar da IX Regiāo Administrutiva, Rio de Janeiro: estudo da população coberta $e$ análise operacional de um subprograma. Rev. Saúde públ., S. Paulo, 9:441-54, 1975

RESUMO: Foi realizada uma avaliação preliminar do Serviço de Saúde Escolar da IX Região Administrativa do Estado do Rio de Janeiro, como resultado do convênio firmado entre o Instituto de Medicina Social da Universidade do Estado do Rio de Janeiro e a Secretaria de Saúde do Estado. Através de estudo do nivel de saude entre os escolares e da análise operacional do subprograma de Registro de Saude, diagnosticaram-se elevados indices de morbidade e pequena proporção de crianças com assistência médica prévia, além de determinar-se a efetividade do programa e de identificar-se problemas no processo técnico-administrativo do Serviço. Dentre as conclusōes destaca-se a necessidade de completar a avaliação do Serviço, através da determinação dos critérios de eficiência, eficácia e rendimento, tendo em vista o fornecimento de subsidios para as tarefas de planejamento e administração. en. fatizada a necessidade de ampliação das medidas integradoras dos serviços universitários com os serviços da comunidade, no sentido de fornecer aos primeiros as condições de realizar, através de prestação de serviços, uma formação dos recursos humanos de acordo com a situação de saúde da comunidade, $e$ aos segundos a necessária implementação de novas técnicas e modelos de assistência.

Unitermos: Serviço de Saúde Escolar, avaliação. Saúde escolar. Saúde pública.

* Trabalho realizado com apoio financeiro e técnico da Organização Panamericana da saúde, Organização Mundial da Saúde. Fundação Kellogg e Fundação Ford.

* Do Instituto de Medicina Social da Universidade do Estado do Rio de Janeiro - Rua Teodoro da Silva, $48-5 . .^{\circ}$ andar - Vila Isabel - ZC-11 $-20.000-$ Rlo de Janeiro, RJ - Brasil.

*** Do Hospital das Clinicas - Universidade do Estado do Rio de Janeiro - Rlo de Janeiro, RJ - Brasil.

**** Da Seção de Medicina Escolar da IX Região Administrativa do Rio de Janeiro Rio de Janeiro, RJ - Brasil 
GASPAR, E. D. et al. - Avaliação preliminar do Serviço de Saúde Escolar da IX Região Administrativa, Rio de Janeiro: estudo da população coberta e análise operacional de um subprograma. Rev. Saúde públ., S. Paulo, 9:441-54, 1975.

No início de 1974, a Universidade do Estado do Rio de Janeiro firmou convênio com a Secretaria de Saúde do Estado, passando o Instituto de Medicina Social da Universidade do Estado do Rio de Janeiro (IMS - UERJ) a trabalhar conjuntamente com a Seção de Medicina Escolar da IX Região Administrativa do Estado do Rio de Janeiro.

Na primeira etapa do trabalho procedeu-se à análise crítica do Programa de Saúde Escolar (SE) do Rio de Janeiro ${ }^{11}$, visando tanto o esclarecimento sobre as proposições de objetivos e atividades do mesmo, como a uma compreensão mais profunda das questões que, há algum tempo, vêm se levantando a respeito de atividades tradicionais da SE.

A análise destaca a uniformidade com que hoje em dia se preconiza a implantação de programas de saúde escolar somente em um contexto de programação geral de saúde infantil e de toda a população.

Sendo objetivo básico de um programa de saúde a melhoria das condições de vida dos indivíduos cobertos, a SE pretende atuar no sentido de melhorar a capacidade da criança cumprindo as características fundamentais do período escolar, as quais são: a aprendizagem, ou seja, o aproveitamento máximo das atividades realizadas na escola visando a um preparo geral ou profissional; e a socialização, isto é, a capacidade de relacionamento com o grupo. Uma criança doente e debilitada, além dos gastos imediatos que seu tratamento requer, bem como os ônus adicionais à rede escolar, por conta das faltas e repetições de ano que cada vez mais sobrecarregam o sistema escolar de $10^{\circ}$ grau (a média nacional de retenções oscila em torno de $42 \%{ }^{1}$ ), possivelmente também terá problemas no decorrer de toda a vida adulta.

A dimensão da população infantil em nosso país justifica o desenvolvimento de programas especiais visando esta faixa etária. A SE tem a vantagem de poder controlar facilmente o grupo a que se destina pelo fato do mesmo estar referido a uma instituição ?.

Considera-se que a SE não consegue cumprir grande parte de seus objetivos por falta de recursos e falhas administrativas. Até que ponto, no entanto, os recursos estão sendo aplicados segundo um planejamento que tenha levado em conta uma avaliação das atividades tradicionalmente desenvolvidas pelo serviço?

Investigando os vários campos de atividades da SE, Rogers e Resse, citados pela Oficina Sanitária Panamericana ${ }^{9}$, concluíram que além do exame físico de rotina não detectar enfermidades que não houvessem sido previamente diagnosticadas, concordavam em que o ausentismo e as consultas médicas de escolares seriam principalmente devidos a problemas emocionais e sociais, e não exatamente a "doenças" que requeressem cuidados médicos.

0 próprio médico-chefe do Departamento de Educação e Ciências de Londres, em seu relatório de 1969-70 ${ }^{4}$, questiona a efetividade e o rendimento do exame médico periódico como instrumento de inspeção de saúde. "Será que a produção em termos de defeitos descobertos justificam o tempo dispendido, ou pode-se executar a tarefa de outra maneira mais efetiva?" O relatório conclui propondo um modelo de inspeção constituído de exame médico de admissão, visitas de seguimento nas escolas, exames médicos subseqüentes quando requisitados - aplicação de questionários aos familiares e procedimentos de "screenings" (testes de acuidade visual e auditiva, medidas de peso e altura).

Segundo artigos publicados pelo Childrens' Bureau Publ. e pelo JAMA, citados por Meyerstein", "muitas pessoas sentem que a maioria dos programas de saúde 
GASPAR, E. D. et al. - Avallação preliminar do Serviço de Saúde Escolar da IX Região Administrativa, Rio de Janeiro: estudo da população coberta e análise operacional de um subprograma. Rev. Saúcle públ., S. Paulo, 9:441-54, 1975.

escolar necessitam ser revistos quanto ao seu conteúdo, procedimentos e objetivos. Largas somas de recursos financeiros são gastas nestes serviços e com efetividade variável. Não foi dada a suficiente importância às mudanças em nossos problemas de saúde, que tiveram diminuídas as frequêencias das doenças transmissíveis e de outras condições agudas". Segundo Wheatley, citado por Meyerstein ${ }^{8}$, muitas leis devem ser modificadas ou até revogadas, como por exemplo aquelas que requerem exame médico periódico anual de todos os escolares. Estes são alguns exemplos de questões que se vêm levantando sobre as atividades da SE.

É evidente que como subsídio a qualquer questionamento é necessário o conhecimento da situação real dos programas. Esta foi a razão porque, além do diagnóstico da situação de saúde do escolar da IX RA, incluiu-se como objetivo desta pesquisa a realização de uma avaliação operacional do serviço de SE. Um sistema de avaliação é essencial para a programação, ou seja, para permitir ao administrador antecipar-se cientificamente aos acontecimentos, com o intuito de controlar e canalizar os múltiplos fatores e circunstâncias que intervêm no decorrer do tempo e que condicionam o alcance dos objetivos propostos.

L'ma avaliação completa deve abranger critérios como: efetividade (relação entre objetivos alcançados e objetivos propostos); eficiência (relação entre utilização efetiva dos recursos e utilização programada); eficácia (relação entre atividades realizadas e atividades programadas); e rendimento (razão entre objetivos alcançados e recursos utilizados) ${ }^{2}$. Pelas características desta investigação, realizada numa etapa inicial do desenrolar do convênio, se pretende a avaliação preliminar de um subprograma, o Registro de Saúde, que permita a identificação de alguns problemas e facilite um diagnóstico da situação do serviço de $\mathrm{SE}$ da IX RA.
DESCRIÇÃO DAS ATIVIDADES DA SEÇAO

DE SACDE ESCOLAR DA IX REGIAO ADMINISTRATIVA DO RIO DE JANEIRO

As atividades definidas para as seções de SE, conforme o Plano de Trabalho elaborado pela Coordenação Geral de Saúde Pública da Secretaria de Saúde do Rio de Janeiro ${ }^{10}{ }^{11}$, estão distribuídas em dois níveis:

\section{A - Atividades de prevenção primária}

1. Palestras de educação sanitária para pais e professores.

2. Verificação das condições sanitárias das escolas.

B - Atividades de prevenção secundária (diagnóstico e correção de danos)

1. Registro de Saúde dos alunos matriculados na rede de ensino estadual. Constam deste registro: certificado de vacinação antivariólica e tríplice (ou dupla); resultado do PPD; exame de acuidade visual; antecedentes pessoais e familiares; exame odontológico e clínico. As enfermidades encontradas devem ser comunicadas ao responsável para que a criança seja encaminhada a tratamento.

2. Revisão dos alunos que terminam o $10^{\circ}$ ciclo do ensino de $1 .^{\circ}$ grau.

3. Atendimento médico nas escolas ou no posto, a qualquer escolar doente. Geralmente as atribuições de saúde escolar limitam-se ao afastamento das aulas para casos de doenças infecto-contagiosas. 
GASPAR, E. D. et al. - Avaliação preliminar do Serviço de Saúde Escolar da IX Região Administrativa, Rio de Janeiro: estudo da populacaão coberta e análise operacional de um subprograma. Rev. Saúde públ., S. Paulo, 9:441-54, 1975.

4. Levantamento de dados sobre doenças mais importantes (deficiência visual e auditiva, dislexia, tuberculose) e acompanhamento dos casos. Estatística de morbidade e mortalidade escolar.

A seção de SE da IX RA localiza-se em prédio próprio, com 4 consultórios médicos, 2 gabinetes dentários, uma sala de radiologia e instalações administrativas. 0 pessoal é constituído por 6 médicos, 9 dentistas, uma assistente-social, 2 técnicos de radiologia, 4 enfermeiras e 6 auxiliares de enfermagem. Um médico e uma enfermeira constituem uma unidade volante que controla os Registros de Saúde nas escolas, Dois médicos atendem diariamente à rotina de inspeção de saúde no Posto; o Posto da IX RA atende a 17 escolas localizadas em Vila Isabel, Andaraí e Grajaú.

O período previsto para a inspeção de saúde dos alunos novos da região compreende de $1 .^{\circ}$ de março a 30 de junho. De acordo com a previsão de matrículas das escolas, é reservado um período para cada uma, prevendo-se o atendimento de 60 crianças por dia, em 4 horas de trabalho. Findo o prazo da escola, as crianças que não compareceram terão exame remarcado para novo período de atendimento, a partir de julho.

Espera-se que a criança já vá ao Posto com o certificado das vacinas obrigatórias e o resultado do PPD. Uma enfermeira colhe a história familiar e pessoal, fisiológica e patológica; outra procede ao exame de acuidade visual. Depois a criança é encaminhada ao exame odontológico e clínico. Todos esses dados são anotados em ficha única (Anexo). Sendo encontrada alguma anormalidade, o médico deve recomendar ao responsável que procure serviço adequado ao tratamento. A SE não tem ligação direta com nenhum outro serviço assistencial.
As recomendações devem ser anotadas no laudo enviado para as escolas, o que teoricamente permite o controle da realização do tratamento.

AVALIAÇÁ DO PROGRAMA DE REGISTRO DE SACDE: METODO

Com o objetivo de realizar o diagnóstico das condições de saúde escolar e avaliar a eficiência do trabalho da SE em identificar os danos, elaborou-se um programa de atividades distribuídas em duas etapas:

$1^{a}$ etapa - Diagnóstico do nível de saúde.

1. Grupo a ser estudado: crianças que ingressaram em 1974, nas escolas da rede estadual da IX RA (matrículas novas).

2. Verificação do número de crianças atendidas no período da primeira chamada para inspeção de saúde, feito por consulta à estatística diária da SE. Somente este grupo foi acompanhado na investigação.

3. Quantificação e classificação dos danos anormais encontrados. Foi feita consulta à estatística diária do serviço (constituida pelas observaçôçes médicas na parte da ficha reservada às recomendações) comparando-a ao levantamento de todas as anotações feitas na ficha de cada criança (história, exame, recomendações). As condições anormais encontradas foram classificadas segundo o comprometimento de aparelhos e sistemas. Procedeu-se da mesma maneira com situaçães que embora não qualificáveis como doenças ou danos, justificavam o controle da criança (por exemplo: PPD forte reator). Grupos com distúrbios neurológicos e psicológicos foram dificilmente divididos, devido 
GASPAR, E. D et al. - Avaliação preliminar do Servjço de Saúde Escolar da IX Região Administrativa, Rio de Janeiro: estudo da população coberta e análise operacional de um subprograma. Rev. Saúde públ, S. Paulo, 9:441-54, 1975.

à subjetividade do exame e superposição no registro de sinais e sintomas.

\section{2a etapa - Avaliação do serviço de saúde} escolar.

Testa etapa tentou-se estabelecer relações entre o atendimento da SE. o cumprimento das orientações fornecidas e algumas variáreis intervenientes. Neste sentido desenvolveram-se as seguintes atividades:

1. Do universo constituído por todas as crianças matriculadas em 1974 que apresentaram alguma anormalidade ao exame para o Registro de Saúde, selecionou-se um grupo constituído por todas as crianças que apresentavam uma mesma condição anormal, isolada ou associada a outras. A "condição índice" a ser selecionada para a avaliação deveria atender aos seguintes critérios: a) ter alta preralência no grupo examinado; $b$ ) contribuir significativamente como causa de dificuldades de aprendizagem; cl possuir critério padronizado de diagnóstico. A condição que melhor atendeu a estes três critérios foi a deficiencia risual, obserrada em 217 escolares.

2. Conrocação. por intermédio das professoras, das crianças que tiveram anotada em sua ficha de Registro de Saúde referência à diminuição de acuidade risual. Convocação dos responsáveis pelas crianças. para se investigar o cumprimento da orientação dada pelo médico. a correção do déficit de acuidade visual detectado no $10^{\circ}$ exame e os fatores que exriceram influência nessas condutas.

3. Verificação. por meio de entrevista. da ocorrência ou não de fatores capazes de influenciar no cumprimento da orientação fornecida aos responsável pela criança como:
- observação médica prévia;

- reforço da professora no sentido do cumprimento da orientação;

- sintomas:

- tipo de encaminhamento.

As hipóteses nulas a serem testadas constituem-se, portanto. na não existência de associação entre os quatro fatores citados e o cumprimento da orientação fornecida.

4. Revisão do exame de acuidade visual nas crianças convocadas, pelo mesmo teste com optotipos de Snellen. utilizado na época do Registro de Saúde.

5. Determinação da efetividade do programa, relacionando-se os objetivos alcançados com os objetivos propostos. ou seja, as crianças que cumpriram a orientação e receberam atendimento especializado com as que receheram orientação.

\section{RESLLTADOS}

Diagnóstico do nivel de saúde

No início de 1974. havia. nas 17 escolas da IX RA, 3.240 matrículas novas. A relação das crianças distribuídas pelas escolas foi encaminhada à seção de SE para que esta elaborasse seu calendário de atividades visando atender o maior número de crianças por ocasião da primeira chamada para o registro de saúde. Verificou-se que das 3.210 crianças matriculadas. $1.018(31.7 \%)$ compareceram para o registro de saúde com uma rariação percentual de zero a $100{ }^{\prime} c$ de comparecimento dependendo da escola.

Segundo o Serviço de Estatística da Seção de SE. destas 1.018 crianças. 157 $(15.4 \%)$ tiveram pelo menos um dano diagnosticado.

Procedendo-se a uma revisão mais acurada nas fichas de todas as crianças atendidas (1.018) e anotando-se além dos dados referidos no item de "recomendações médicas" os referidos nos itens de 
GASPAR, E. D. et al. - Avallação preliminar do Serviço de Saúde Escolar da IX Reglāo Administrativa, Rio de Janeiro: estudo da populaça coberta e análise operacional de um subprograma. Rev. Saúde públ., S. Paulo, 9:441-54, 1975.

vacinações, antecedentes e exame médico, chegou-se a um total de 707 crianças com algum dano diagnosticado, correspondendo a $69,4 \%$ do grupo examinado.

Destas 707 crianças, $283(40 \%)$ tinham um dano diagnosticado, $211 \quad(29,8 \%)$ dois danos, $125(17,6 \%)$ três, $89(12,5 \%)$ quatro e mais (Tabela 1).

\section{TABELA 1}

Frequiencia de crianças com danos segundo o registro de saúde de 1.018 crianças da IX Regiáo Administrativa da Secretarla da Saúde do Estado do Rio de Janeiro (IX RA - 1974)

\begin{tabular}{|c|c|c|c|}
\hline \multirow{2}{*}{$\begin{array}{l}\text { Número } \\
\text { de danos }\end{array}$} & \multicolumn{3}{|c|}{$\begin{array}{c}\text { Freqüência de crianças } \\
\text { com danos }\end{array}$} \\
\hline & N.o & $\%$ & $\begin{array}{l}\text { acumu- } \\
\text { lada }\end{array}$ \\
\hline 1 & 283 & 27,7 & 27,8 \\
\hline 2 & 211 & 20,7 & 48,5 \\
\hline 3 & 125 & 12,2 & 60,8 \\
\hline 4 e mais & 89 & 8,7 & 69,5 \\
\hline $\begin{array}{l}\text { Nenhum } \\
\text { Não }\end{array}$ & 241 & 23,6 & 93,2 \\
\hline encontrado & 69 & 6,7 & 100,0 \\
\hline Total & 1.018 & 100,0 & - \\
\hline
\end{tabular}

As condições anormais encontradas foram classificadas e resultaram onze grupos de danos com uma freqüência variando de $1,7 \%$ - grupo de "outras condições" - até 18,7\% - danos neurológi$\cos$ (Tabela 2).

\section{Avaliação do Registro de Saúde}

Nesta segunda etapa do estudo procedeu-se à determinaçấo da efetividade do programa e à investigação sobre as relações das variáveis com o cumprimento das recomendações.

a) Determinação da efetividade do programa.

Das 217 crianças com diagnóstico de acuidade visual deficiente, convocadas para novo exame e entrevista com os responsáveis, compareceram $166 \quad(76,5 \%)$.

No grupo entrevistado verificou-se que 114 crianças $(68,6 \%)$ não receberam encaminhamento para serviço de assistência médica e das outras 52 crianças, 21 $(41,1 \%)$ foram encaminhadas para o INPS, $20(38,4 \%)$ para serviço não especificado, $7(13,4 \%)$ para consultórios ou serviços particulares, $3(5,7 \%)$ para o Hospital de Clínicas da UERJ e uma não informou a respeito.

Destes 51 encaminhamentos, $42(82,3 \%)$ foram verbais e $9(17,6 \%)$ por escrito.

Do total de 51 que informaram ter recebido encaminhamento, $18 \quad(35,3 \%)$ cumpriram a recomendação, e destas, 15 estavam em tratamento por ocasião da entrevista, o que configura uma efetividade de $9 \%$ (objetivos alcançados/objetivos propostos $=15 / 166=9 \%$ ). Das restantes 33 que não cumpriram a recomendação, as argumentaçôes foram várias: a mais frequiente foi "falta de tempo",

TABEL 2

Distribuição da frequência de danos encontrados em 1.018 criancas atendidas * na primeira chamada para registro de saúde segundo grupos de danos (IX RA - 1974)

\begin{tabular}{|c|c|c|}
\hline \multirow[t]{2}{*}{ Grupos de danos } & \multicolumn{2}{|c|}{$\begin{array}{c}\text { Freqüência de } \\
\text { danos }\end{array}$} \\
\hline & N.o & $\%$ \\
\hline Neurológicos & 253 & 18,7 \\
\hline Oftaimológicos & 227 & 16,8 \\
\hline Otorrinolaringológicos & 191 & 14,1 \\
\hline Digestivos & 175 & 12,9 \\
\hline Desenvolvimento & 132 & 9,7 \\
\hline Anomalias fisicas & 106 & 7,8 \\
\hline Respiratórios & 93 & 6,8 \\
\hline Geniturinários & 67 & 4,9 \\
\hline Psicológicos & 53 & 3,9 \\
\hline Dermatológicos & 31 & 2,2 \\
\hline Outras condiçōes & 23 & 1,7 \\
\hline Total & 1.351 & 100,0 \\
\hline
\end{tabular}

* Muitas crianças estão contadas mals de uma vez por apresentarem vários danos. 
GASPAR, E. D. et aI. - Avaląão preliminar do Serviço de Saúde Escolar da Ix Região Administrativa, Rio de Janeiro: estudo da população coberta e análıse operacional de um subprograma. Rev. Saúle piubl., S. Paulo, 9:441-54, 1975.

seguida de "dificuldades econômicas", "problema sem importância" "descuido", "outros afazeres".

Procedido o reexame das 166 crianças com a mesma técnica de "screening", verificou-se a confirmação de deficiente acuidade visual em 89 crianças havendo, portanto. $53.6 \%$ de concordância entre o primeiro e o segundo exames. Se considerarmos este grupo como sendo o que realmente sofria de deficiência risual, a efetividade do programa passa a ser de $16,8 \%$ c $(15 / 89)$.
b) Variáveis relacionadas ao cumpri- mento das recomendações.

Dos 166 pais que compareceram, 20 $(11.5 \%$ ) referiram ter recebido recomendações da professora para procurar assistência médica devido ao problema visual da criança e $34(20.5 \%)$ informaram positivamente sobre a existência de observação médica prévia. Verificou-se que 108 $(65 \%)$ dos laudos do Registro de Saúde, arquivados nas escolas, estaram em branco. em 12 constava o diagnóstico de dé-

T A BEL 3

Relaçāo entre observação médica prévia e cumprimento da orientação da $\mathrm{SE}$ em 51 * crianças com acuidade visual deficiente (IX RA - 1974)

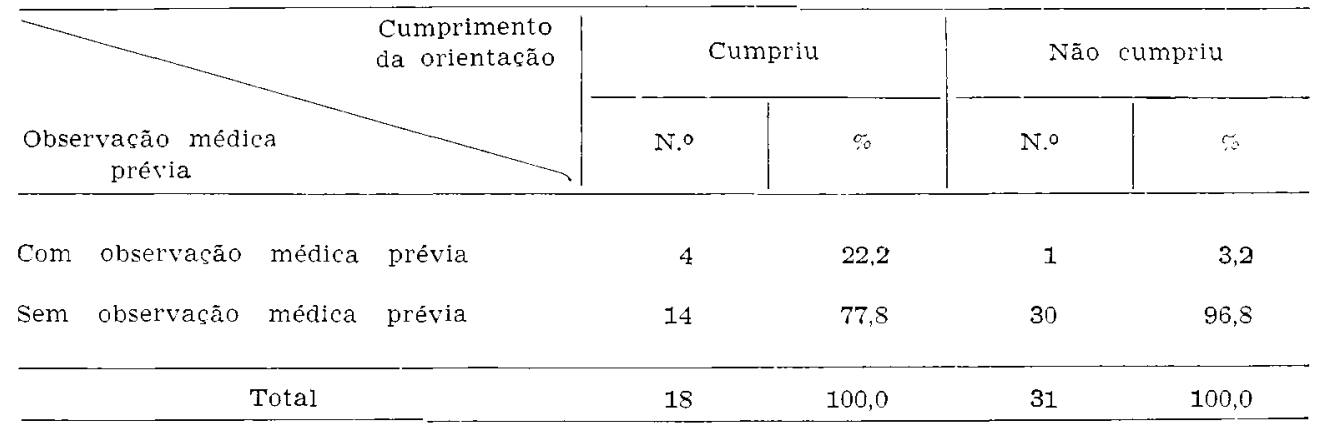

* Duas crianças não informaram a respeito.

$$
\chi^{2}=2,651 \text { (com correşão de Yates) } \quad p=\text { N.S. }
$$

ficit visual e em 18 estava referido encaminhamento a oftalmologista.

Das 51 crianças que receberam encaminhamento. 5 tinham referência à observação médica prévia constatando deficiência visual e destas. 4 cumpriram o encaminhamento (Tabela 3); 12 tinham referência à recomendação da professora para que efetivassem o encaminhamento, e destas, 6 a cumpriram (Tabela 4 ); 35 apresentaram sintomas, e destas, 14 cumpriram o encaminhamento (Tabela 5); e 35 receberam encaminhamentos específicos. isto é, orientação de qual instituição deveria ser procurada, e destas, 12 cumpriram o encaminhamento (Tabela 6). A aplicação do teste do Qui-quadrado nas Tabelas 4, 5 e 6 e do Qui-quadrado corrigido na Tabela 3 mostrou que as diferenças encontradas não são signiifcativas e. portanto, não se pode afirmar que haja associações entre o ter sido ou não cumprido o encaminhamento e as variáveis intervenientes estudadas. 
GASPAR, E. D. et al. - Avaliação preliminar do Serviço de Saúde Escolar da IX Região Administrativa, Rio de Janeiro: estudo da população coberta e análıse operacional de um subprograma. Rev. Saúde públ., S. Paulo, 9:441-54, 1975.

\section{T A BELA 4}

Relação entre reforço da professora e cumprimento da orientação da SE em $51 *$ crianças com acuidade visual deficiente (IX RA - 1974)

\begin{tabular}{|c|c|c|c|c|}
\hline \multirow[b]{2}{*}{$\begin{array}{c}\text { Reforco da } \\
\text { professora }\end{array}$} & \multicolumn{2}{|c|}{ Cumpriu } & \multicolumn{2}{|c|}{ Não cumpriu } \\
\hline & N. ${ }^{\circ}$ & $\%$ & N.O & $\%$ \\
\hline Com reforco & 6 & 35,3 & 6 & 18,7 \\
\hline Sem reforco & 11 & 64,7 & 26 & 81,3 \\
\hline Total & 17 & 100,0 & 32 & 100,0 \\
\hline
\end{tabular}

Duas crianças não informaram a respeito.

$$
x^{2}=1,697 \quad \mathrm{p}=\text { N.S. }
$$

TA B E A 5

Relação entre sintomatologia e cumprimento da orientação da SE em 51 crianças com acuidade visual deficiente (IX RA - 1974)

\begin{tabular}{|c|c|c|c|c|}
\hline \multirow[b]{2}{*}{ Sintomatologia } & \multicolumn{2}{|c|}{ Cumpriu } & \multicolumn{2}{|c|}{ Não cumpriu } \\
\hline & No & $\%$ & N.o & $\%$ \\
\hline Com sintomatologia & 14 & 77,8 & 21 & 63,6 \\
\hline Sem sintomatologia & 4 & 22,2 & 12 & 36,4 \\
\hline Total & 18 & 100,0 & 33 & 100,0 \\
\hline
\end{tabular}

\section{DISCUSS Ã O}

A primeira observação é sobre a elevada percentagem de faltas à inspeção de saúde na primeira chamada $(68,3 \%)$, o que permite questionar o rendimento do programa (razão entre objetivos alcançados e recursos utilizados). Os índices de comparecimento variaram nas diferentes escolas. Supõe-se que as diferenças são devidas tanto à disponibilidade de um adulto para levar a criança ao exame, como à percepção que os pais têm da ameaça à saúde e do valor daquela conduta preventiva ${ }^{3}$. Não fez parte do estudo a investigação desse problema. 
GASPAR, E. D. et al. - Avaliação preliminar do Serviço de Saúde Escolar da IX Região Administrativa, Rio de Janeiro: estudo da população coberta e análise operacional de um subprograma. Rev. Saúde públ., S. Paulo, 9:441-54, 1975.

TABELA 6

Relação entre tipo de encaminhamento e cumprimento da orientação da SE para 51 crianças com acuidade visual deficiente (IX RA - 1974)

\begin{tabular}{l}
$\begin{array}{c}\text { Cumprimento } \\
\text { da orientação }\end{array}$ \\
$\begin{array}{l}\text { Tipo de } \\
\text { encaminhamento }\end{array}$ \\
\hline $\begin{array}{l}\text { Específico } \\
\text { Inespecifico }\end{array}$ \\
\hline Total
\end{tabular}

Lima alternativa que provavelmente reduziria parte desse problema seria a realização dos exames nas escolas. Isto facilitaria o controle das crianças, aumentaria a eficiência dos recursos disponíveis e eliminaria os longos períodos de segunda chamada, permitindo a realização de outros programas.

Outro fato importante, que certamente influencia a programação da SE é o sub-registro do serviço de estatística $(550 / 707=78 \%)$, devido principalmente à maneira subjetiva como é preenchida a ficha individual, e à ausência de espaços específicos para resumo de diagnóstico, recomendaçôes e encaminhamento. 0 preenchimento das fichas não parece depender da importância da enfermidade, mas está relacionado possivelmente ao treinamento do médico.

Verificou-se que uma percentagem semelhante de crianças é aparentemente normal $(23.6 \%)$, ou apresenta um ou dois danos $(27,3 \%$ e $20,3 \%)$. Menor número de crianças apresentavam três ou mais lesões. Estas percentagens não nos fornecem uma relação com a relevância do dano. Assim, uma criança pode ter três danos leves, e outra um dano grave que force a procura do médico. Mas o aspecto importante é realçar que, em média, $70 \%$ das crianças examinadas apresentavam algum problema de saúde. Por este estudo não se pode esclarecer se o fato do médico indicar tratamento para várias doenças reforça a procura de atendimento posterior pela família.

$\mathrm{Na}$ análise dos danos encontrados, durante a tentativa de agrupamento por doenças, enfrentou-se um obstáculo: a su'wjetividade e a diversidade de critérios para a realização dos exames físicos, dos testes e mesmo do preenchimento da ficha de Registro de Saúde. Os testes, pela importância que representam como instrumentos para se chegar aos diagnósticos, e pelas características que assumem (baixo custo, relativamente simples e de fácil aceitação) devem ser sensiveis (dando pequena proporção de resultados falso-negativos) e, se possível, também específicos (dando pequena proporção de resultados falso-positivos) ${ }^{12}$. Reunindo-se vários sinais e sintomas sugestivos de distúrbios neurológicos, estes constituíram o maior grupo de danos $(18,7 \%)$; mas o limite entre os distúrbios neurológicos e os psíquicos é pouco nítido, de acordo com os 
GASPAR, E. D. et al. - Avaliação preliminar do Serviço de Saúde Escolar da IX Região Administrativa, Rlo de Janeiro: estudo da população coberta e análise operacional de um subprograma. Rev. Saúde públ., S. Paulo, 9:441-54, 1975.

termos utilizados pelo médico e com as diferentes definiçóes ligadas a cada termo. Além da indefinição de várias enfermidades encontradas, possivelmente também ocorre outro falseamento dos resultados: a falta de uma rotina objetiva de exame implica provável subestimação dos diagnósticos. Exemplos disso são: a verminose que tem vários critérios de diagnóstico ou suposição - exame de fezes positivo, queixa de que a "criança tem ou põe vermes", anorexia, diarréia, dor abdominal, etc.; a desnutrição, em relação à qual existem vários estudos mostrando que uma grande parte da população infantil do nosso meio está situada na faixa da distrofia leve, assintomática ou oligossintomática.

Estas observações demonstram a importância da elaboração de rotinas objetivas para exame e, o mais importante, o treinamento de todos os médicos nessa rotina. Justifica-se também a existência de uma rotina assistencial, devido ao grande número de danos à saúde, que influenciam no aproveitamento escolar, e que são em muitos casos, de correçáo relativamente fácil. Neste ponto, nossas observaçóes diferem das feitas por autores estrangeiros que concluíram que as consultas à $\mathrm{SE}$ geralmente não eram feitas por problemas essencialmente médicos. Em nosso meio existem problemas como a verminose e a desnutrição que, apesar de terem sua solução muito mais na dependência de uma melhoria do nível sócio-econômico das comunidades, ensejam a atuação integrada da SE com outros setores da população.

Apesar dos esforços realizados no sentido de reduzir ao mínimo a proporção de não-participantes - considera-se razoável de 5 a $10 \%^{12}$ - conseguiu-se o comparecimento, após duas e até três visitas às escolas, de $166(76,5 \%)$ das 217 crianças com deficiência visual diagnosticada no Registro de Saúde. $\mathbf{O}$ fato de que destes, somente $20,5 \%$ referiam ter recebido alguma observação médica prévia, reforça a importância do serviço de SE em nosso meio.

$O$ fato de que no reexame das crianças, por ocasião da entrevista com o responsável, ter sido confirmada a deficiência de acuidade visual em somente 89 das 166 crianças examinadas, compele a discussão em torno da sensibilidade e especificidade do teste utilizado, ou seja, dos optotipos de Snellen. Justifica-se um estudo da confiabilidade da técnica. Aliás se propõe uma avaliação dos testes usados em "screenings" antes de serem introduzidos na rotina do serviço médico ${ }^{5,8}$, pois os mesmos devem satisfazer critérios de sensibilidade, precisão e especificidade, além de serem economicamente justificáveis no contexto de financiamento dos serviços de saúde.

O objetivo de avaliar a hipótese de que o encaminhamento por escrito e específico aumentaria o índice de cumprimento das medidas ficou prejudicado pelo pequeno número encontrado, ou seja, 9 encaminhamentos por escrito e 42 de forma verbal. Ressalte-se o fato de que das 166 crianças com deficiência visual diagnosticada no Registro de Saúde, somente $52 \quad(31,3 \%)$ receberam algum tipo de encaminhamento, e destas, 18 procuraram assistência médica especializada.

A tentativa de se estabelecer associaçōes entre o atendimento da SE, o cumprimento das orientações recebidas e a influência de algumas variáveis como a recomendação da professora, a presença de sintomas, a existência de observação médica prévia e o encaminhamento específico, resultou, após a aplicação dos testes estatísticos (qui-quadrado nas Tabelas 4,5 e 6 e qui-quadrado corrigido na Tabela 3 , todas com um grau de liberdade e fixando-se o nível de significância em $\mathrm{p}=0,05)$, na aceitação das hipóteses 
GASPAR, E. D. et al. - Avaliação preliminar do serviço de Saúde Escolar da IX Região Administrativa, Rio de Janeiro: estudo da população coberta e análise operacional de um subprograma. Rev. Saúde públ., S. Paulo, 9:441-54, 1975.

nulas, ou seja, na inexistência de associação positiva.

\section{CONCLUSOES}

1. E elevada a morbidade entre os escolares da IX RA e baixa a percentagem de crianças cujos danos já têm diagnóstico e tratamento prévio, o que reforça a importância, em nosso meio, do papel a ser desempenhado pela SE. Deve-se discutir a ampliação das atribuições da SE no sentido de prestar outros serviços assistenciais além de diagnósticos, já que grande parte das enfermidades observadas é de fácil controle ambulatorial. Além disso, deve-se pensar na dispersão causada pelo encaminhamento das crianças de um para outro serviço médico. Esta prática, além de levar a uma duplicação indesejável de recursos, dificulta o tratamento da criança, pela necessidade de múltiplos deslocamentos e maiores gastos.

2. Pela importância que representam as estatísticas de morbidade para as tarefas de planejamento de atividades e pela ocorrência do sub-registro determinada no estudo, impõem-se medidas que aprimorem o serviço, quer ao nivel de reestruturação da ficha de exame (no sentido de torná-la mais objetiva quanto ao diagnóstico e tratamento), quer ao nivel da própria rotina do exame.

3. É elevado o índice de não comparecimento à sede da seção de $\mathrm{SE}$ por ocasião da primeira chamada do exame de Registro de Saúde, o que reforça a necessidade de se testar a realização do Registro de Saúde por equipe volante.

4. Apesar das professoras serem frequentemente indicadas como importantes agentes de saúde na comunidade, o estudo demonstrou que está havendo pouca preo- cupação pelo seu aproveitamento, pois a comunicação através dos laudos é negligenciada (65\% em branco). Além dessa etapa importante - a informação também o esclarecimento e a orientação das professoras não são realizados.

5. O fato de $41,1 \%$ dos encaminhamentos específicos terem sido feitos para o INPS, reforça a necessidade de coordenação com os serviços assistenciais, visando possibilitar o encaminhamento direto das crianças.

6. As diferenças existentes entre os resultados obtidos pela aplicação do teste com optotipos, na detecção de déficits na acuidade visual, justificam a necessidade de se programar um estudo avaliando sua confiabilidade.

7. Os resultados deste estudo, de caráter preliminar, enfatizam a necessidade de se proceder a uma avaliação completa do serviço de SE, incluindo os critérios de efetividade, eficiência, eficácia e principalmente rendimento.

8. Na medida em que dispomos destes resultados, relativos a um serviço de SE que conta com razoáveis recursos e atende a uma população de regular nível sócio-econômico como é o da IX RA, pode-se estimar a situação dos demais serviços de SE do Estado e das suas respectivas populações escolares.

9. Este trabalho realça a importância dos convênios entre as universidades e os serviços de saúde da rede estadual, possibilitando não apenas o treinamento de pessoal em atividades concretas de saúde, como levando o instrumental desenvolvido pela universidade para a implementação das tarefas rotineiras. 
GASPAR, E. D. et al. - Avaliação preliminar do Serviço de Saúde Escolar da IX Regiáo Administrativa, Río de Janeiro: estudo da população coberta e análise operacional de um subprograma. Rev. Saúde pübl., S. Paulo, 9:441-54, 1975.

\section{RSPU-B/283}

GASPAR, E. D. et al. - [Preliminary evaluation of State of Rio de Janeiro's Ninth Administrative Region's School Health Service: study of the covered population and operational analysis of a subprogramme]. Rev. Saúde públ., S. Paulo, 9:441-54, 1975.

SUMMARY: A preliminary evaluation of the School Health Service of the State of Rio de Janeiro's Ninth Administrative Region was performed as result of an agreement between the Institute of Social Medicine belonging to the State of Rio de Janeiro University and the State Department of Health. Through an analysis of the school-children's health level and an operational study of the health register subprogramme, high morbidity rates were found as was also a small percentage of children having received previous medical care. The effectiveness of the programme and the identification of problems in the technical and administrative area of the service were determined. Among the results the need to complete the Service's evaluation through the use of the efficacy, efficiency and yielding criteria considering the availability of funds for planning and administration was emphasized. It is important to increase interaction between University and Community services in order to supply the former with conditions that enable the development of human resources adequate to the Community health conditions providing the latter with the necessary implementation of new techniques and patterns of attention.

UNITERMS: School Health Service, evaluation. Public health.

\section{REFERENCIAS BIBLIOGRAFICAS}

1. DIRETORIA do MEC diz que 4 milhões de crlanças de 7 a 14 anos estão sem escola. Jornal da Tarde, Rio de Janeiro, fev. 1974 . p. 13.

2. FERRARA, F. A. et al. - Medicina de la comunidad: medicina preventiva, medicina social, medicina administrativa. Buenos Aires, Inter-Medica, 1972.

3. GARCIA, J. C. - Sociologia y medicina: bases sociologicas de las relaciones medico-paciente. [Washington, D.C., OPAS] 1972. (Antologia de Sociologia Medica - mimeografado).

4. HEALTH screening at school. Lancet, 2 : 1134, 1972.

5. HOLLAND, N. W. - Taking stock. Lancet, 2:1494, 1974.

6. HORWITZ, O. - Long-range evaluation of a mass screening program. Amer. J. Epidem., 100:20-8, 1974.

7. KLOETZEL, K. - As bases da medicina preventiva. São Paulo, Edart, 1973.
8. MEYERSTEIN, A. N. - The value of periodic school health examinations. Amer. J. publ. Hlth., 69:1910-26, 1969.

9. PROGRAMA de salud escolar. Bol. Ofic. Sant. panamer., 58:544-5, 1965.

10. RIO DE JANEIRO. Secretaria de Saúde. Assessoria de Saúde Escolar. Unidade especializada em medicina escolar. Rlo de Janeiro, s.d. (mimeografado).

11. RIO DE JANEIRO. Secretaria de Saúde. Coordenação Geral de Saúde Pública - Ordem de servico " $E$ " GSP 12, de 07/12/1979: estabelece normas para o registro de saude nos Centros de saúde e dá outras providências. Rlo de Janeiro, 1973 (mlmeografado).

12. SZKLO, M. - Inquéritos de morbidade, Rev. Med. Atm., 9:11-24, 1974.

Recebido para publicacão em 26/06/1975 Aprovado para publicação em $22 / 09 / 1975$ 
NOME :

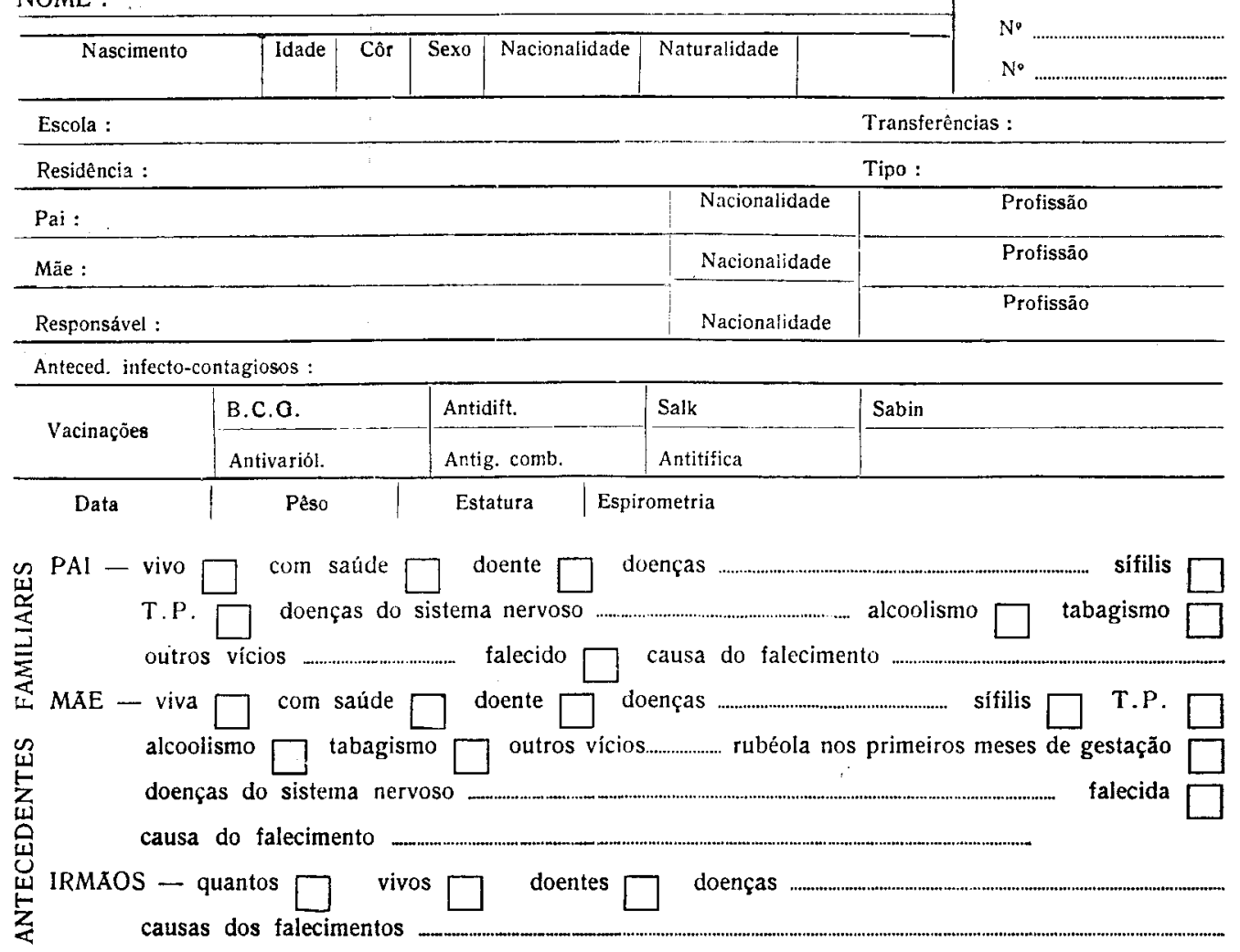

ANTECEDENTES PESSOAIS

Gestação. parto... levantou a cabeça aos meses.

Sentou-se aos...... meses. Engatinhou aos..... meses. Andou aos..... meses. Falou aos..... meses. Chupou dedos 1" dentição aos......... meses. $2^{\mathfrak{a}}$ aos......... meses. $1^{\circ}$ tratamento dentário com........ meses. Defeito da fala fraturas $\square$ alergias $\square$ hospitalizaçōes $\square$ causas .................................................................... canhoto $\square$ surtos reumatimais $\square$ icterícia $\square$ gripes $\square$ diarréias $\square$ ALIMENTAÇAO: $\mathbf{n}^{\circ}$ diário SONO: agitado $\square$ tranquiilo $\square$ sonâmbulo $\square$ insônia $\square$ ㄴ Casa $\square$ apartamento $\square$ barraco $\square$ coletiva $\square$ água corrente $\square$ esgôto $\square$ fossa $\square$

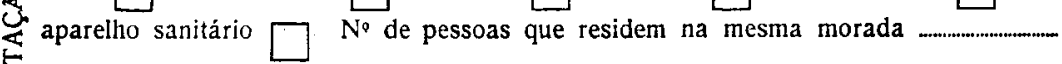

Ass. Enf.:
$\angle A U D O$
$D E \quad S A U D D E \quad E S C O L A R$
R.S. n.

D.M. - ESCOLA : nascido a

filho de tendo como responsável

residente à

submetido à inspeção de saúde escolar, foi considerado para a freqüência escolar.

Rio de Janeiro, GB, de de 19 


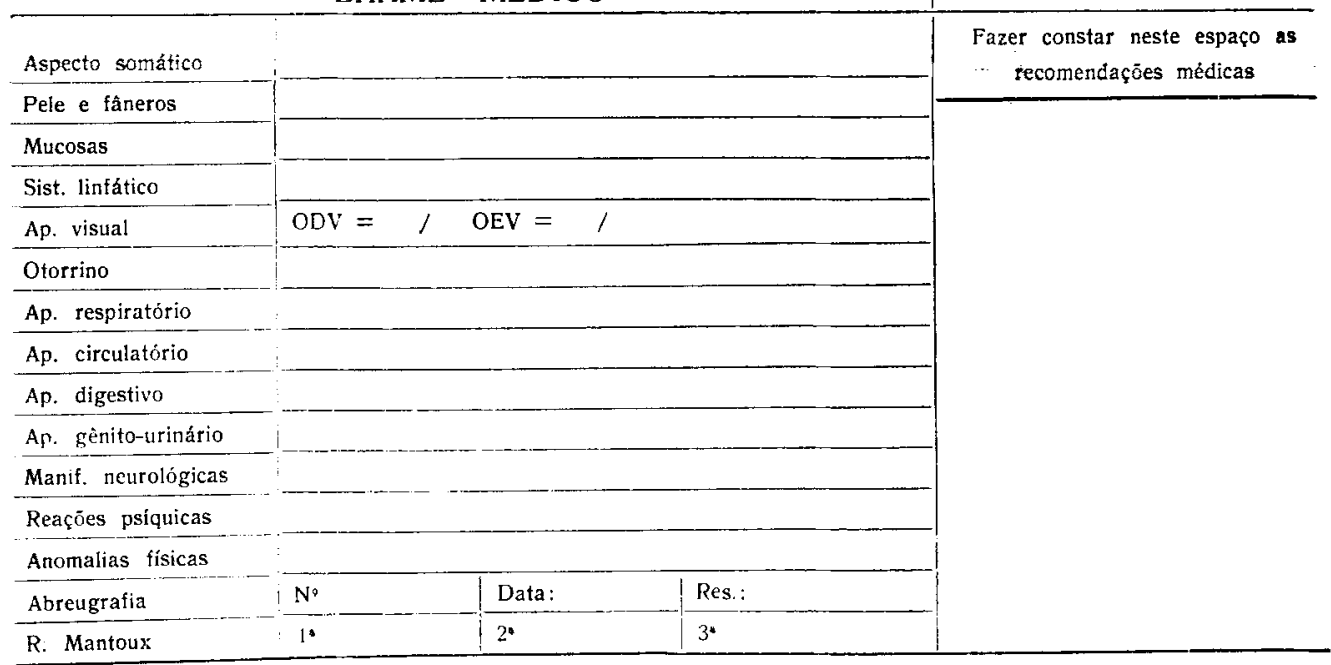

CONCLUSAO:

Data: ................................... 19

\section{EXAME ODONTOLOGICO}

Indice $C P O D$

Levantamento da cárie dentária
OBSERVAÇOES

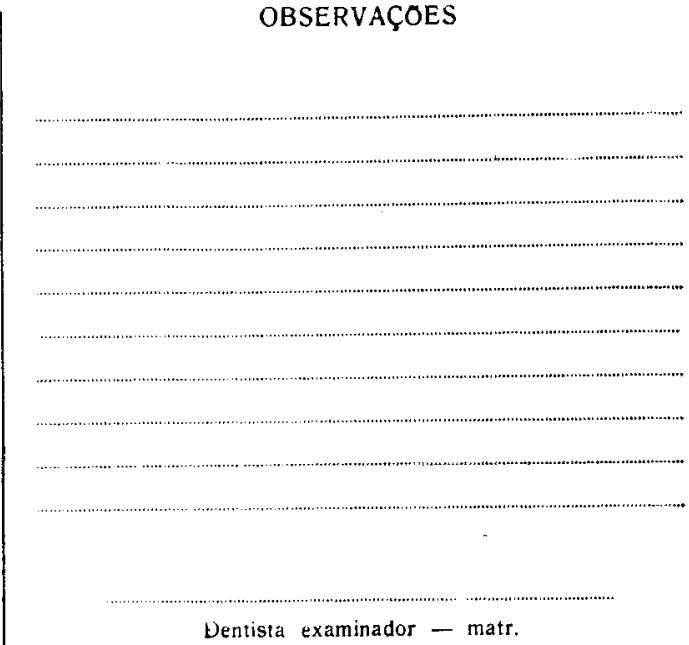

RECOMENDAÇOES

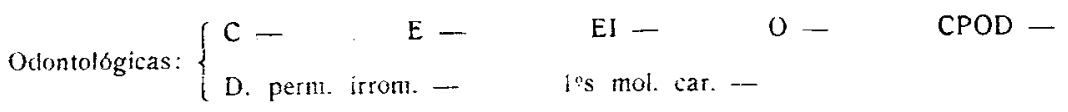

Dentista - matr. 